\title{
Herpes Simplex Encephalitis: A Case Report
}

\author{
David Zahirović ${ }^{1^{*}}$ (D), Ema Dejhalla ${ }^{2}$ (D), Tina Zavidić 3,4 (D)
}

\author{
${ }^{1}$ Primorje-Gorski Kotar County \\ Health Centers, Rijeka, Croatia \\ ${ }^{2}$ Medical Centre for Occupational \\ Health, Rijeka, Croatia \\ ${ }^{3}$ Family Medicine Specialist, Istrian \\ Health Centers, Pazin, Croatia \\ ${ }^{4}$ University of Rijeka, Faculty of \\ Medicine, Rijeka, Croatia
}

\begin{abstract}
Herpes simplex virus encephalitis (HSVE) is an acute or subacute illness associated with focal or global cerebral dysfunction. It is caused by herpes simplex viruses belonging to either type 1(HSV-1) or type 2(HSV-2). Most of HSVE is caused by HSV-1. The most common manifestations include encephalopathy, fever, seizures, headaches, and focal neurological deficits.

49-year-old patient who has diabetes mellitus type 1, first presented with a fever, followed by a generalized epileptic seizure. In the Emergency Room a lumbar puncture was performed, and HSV-1 was detected in the cerebrospinal fluid. MR of the brain concluded that the changes correspond to the changes within HSV-1 encephalitis with a smaller area of hemorrhage. The patient was prescribed with acyclovir $3 \times 10 \mathrm{mg} / \mathrm{kg}$ i.v. for three weeks. After antiviral therapy and glycemic control, the patient was discharged with antiepileptic, antihypertensive, antiulcer and antidiabetic drugs and indication to do a control EEG and infectologist exam with laboratory findings.
\end{abstract}

HSVE has high morbidity and mortality, and the treatment of choice is acyclovir therapy.

Keywords: herpes simplex virus type 1, encephalitis, diabetes mellitus type 1

\section{Correspondence: \\ David Zahirović}

Address: Primorje-Gorski Kotar County Health Centers, Rijeka,

Croatia

Email: davidzahirovic3@gmail.com
Received: 08.02.2022,

Accepted: 21.02.2022

https://doi.org/10.29333/jcei/11831

\section{INTRODUCTION}

Herpes simplex encephalitis (HSVE) is caused by herpes simplex virus type 1 (HSV1) or type 2 (HSV-2) [1]. The vast majority of HSVE is caused by HSV-1 [2]. There are major and minor criteria for diagnosis of encephalitis and encephalopathy [3]. Major is required and includes patients presenting with altered mental status (defined as decreased or altered level of consciousness, lethargy or personality change) lasting $\geq 24$ hours with no alternative cause identified. Minor criteria are fever $\geq 38^{\circ} \mathrm{C}$ within the 72 hours before or after presentation, generalized or partial seizures not fully attributable to a pre-existing seizure disorder, new onset of focal neurologic findings, CSF WBC count $\geq 5 /$ cubic $\mathrm{mm}$, abnormality of brain parenchyma on neuroimaging suggestive of encephalitis that is either new from prior studies or appears acute in onset, abnormality on electroencephalography that is consistent with encephalitis and not attributable to another cause [3]. Two minor criteria are required for possible encephalitis and $\geq 3$ required for probable or confirmed encephalitis. Clinical manifestations of HSVE include prodromal phase of fever, malaise, headache and nausea which often precedes more severe neurologic symptoms like focal cranial nerve palsies, hemiparesis, dysphasia, aphasia, ataxia, visual field defects and papilledema $[4,5]$. None of the clinical features are pathognomonic of HSVE [6]. Cognitive or behavioral changes could be misdiagnosed as a psychiatric disease. Morbidity and mortality are greater if treatment is delayed or inadequate [7].

\section{CASE}

A 49-year-old patient who had been febrile $38.5^{\circ} \mathrm{C}$ for ten days, was admitted to the neurology department as indicated by an infectologist. He was previously treated with $2 \mathrm{~g}$ of ceftriaxone i.v. due to the above symptoms. On the day of admission, the patient had a generalized epileptic seizure at home, after which he was transported to the hospital. The patient did not reconstruct the event. Patient's fever was accompanied by chills and tremors, especially in the evening. 


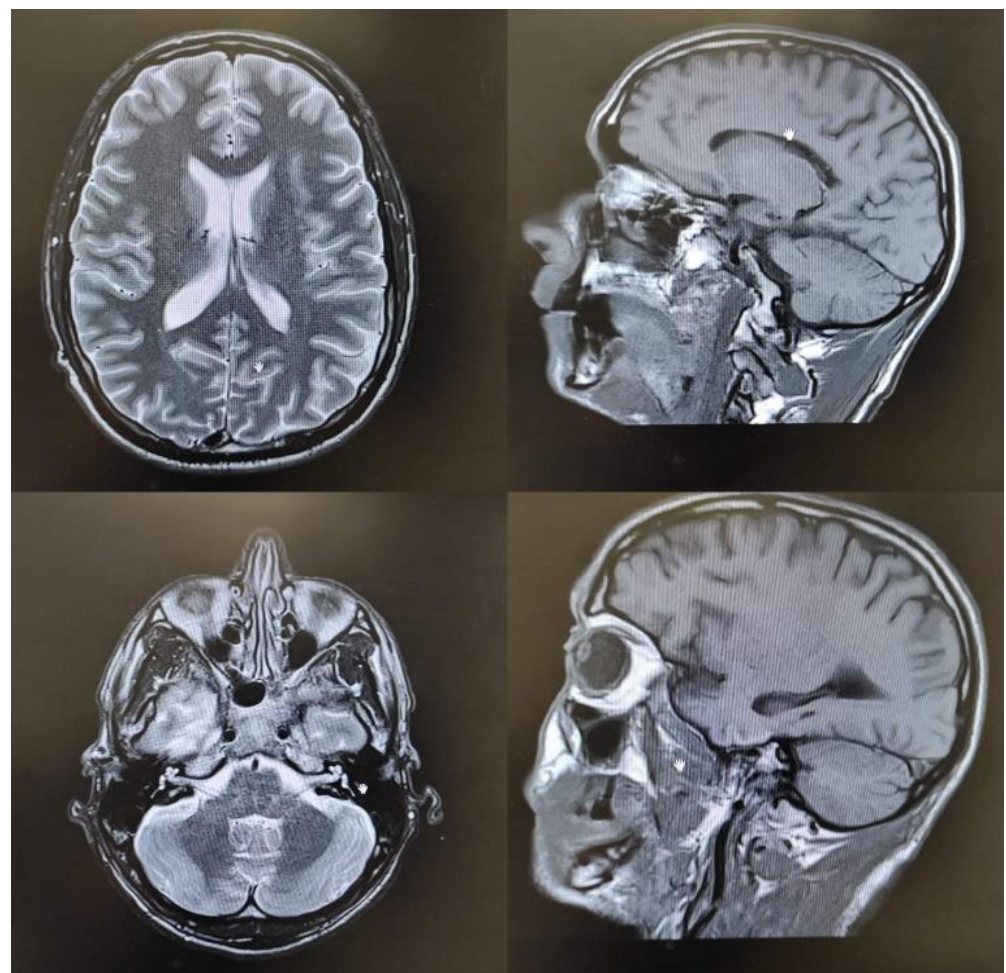

Figure 1. MR of the brain in a 49-year-old patient

Occasionally he had temporal headaches and nausea. He denied vomiting, diarrhea, photophobia, dyspnea, chest pain, abdominal pain, dysuria. Also, the patient denied abusive NSAIDs or paracetamol. He has a diabetes mellitus type 1 and in chronic therapy he has insulin glargine and insulin aspart. He denied drug allergies. During the emergency exam, a lumbar puncture was performed, and patient experienced another epileptic seizure.

At admission, the patient was eupneic, hemodynamically stable with a pressure of $125 / 80 \mathrm{mmHg}$, pulse 90 , skin and mucous membranes were properly circulated, there were no signs of meningism. An enlarged lymph node was seen retromandibularly on the left side. Tonsils weren't enlarged, tongue coated, buccal mucosa without enanthema, tooth untreated. Brain nerve outlets painless. The paranasal sinuses were painless on palpation. Scalp was without changes, there was no discharge from the ear, mastoids were painless. Auscultatory normal lung breathing without accompanying sound phenomena. Heart tones were rhythmic, systolic murmur present $1 / 6$. Abdomen was at chest level, soft, elastic and painless on palpation. Peristalsis audible. Enlargement of intra-abdominal organs was not palpable. Lumbar succussion was negative. Limbs were symmetrical, without edema, palpable peripheral pulsations. There were no new skin changes.

The patient was conscious, oriented in all three directions, neck free, upper and lower Brudzinski sign were negative, Kernig sign was negative, speech without changes. Eye slits were symmetrical, pupils isosceles, medium wide proper reactions to light, bulbs centralized, bulbomotoric skills were normal, no nystagmus. The patient denied binocular or other visual disturbances. Swallowing was orderly. Muscles were with orderly trophism and tone. Rough arm muscle strength maintained. Patient could maintain all four extremities in an antigravity position. Myotatic reflexes were symmetric and appropriate. Plantar reflex showed bilateral plantar flexion. Cutaneous abdominal reflexes were elicited symmetrically in all three grades. Diadochokinesis was in order. Romberg was negative. The patient did not report sensory outbursts. He controlled the sphincters.

Initial laboratory findings showed the following pathological values; leukocytosis $\left(16 \times 10^{9}\right)$, neutrophilia $\left(9 \times 10^{9}\right)$, lymphocytosis $\left(9 \times 10^{9}\right)$, monocytosis $\left(3.4 \times 10^{9}\right)$, hyperglycemia $13.4 \mathrm{mmol} / \mathrm{L}$, creatinine $135 \mu \mathrm{mol} / \mathrm{L}$, eGFR $53 \mathrm{~mL} / \mathrm{min} / 1.73 \mathrm{~m}^{2}$. It should be noted that the CRP (Creactive protein) value was $<0.6$. Acid-base status showed values in favor of metabolic acidosis ( $\mathrm{Ph} 7.2, \mathrm{pCo} 23.8$, $\mathrm{HCO} 3$ 10.8). Glucose and ketones were present in the complete urine analysis. The coagulation tests were within the reference value.

Extensive microbiological treatment was performed, and HSV-1 was detected in the cerebrospinal fluid. Other findings of microbiological treatment did not show positive results. Radiological processing included CT (computed tomography) of the brain, AP (anteroposterior) radiograph of the thoracic organs (findings showed no pathological changes) and MRI (magnetic resonance imaging) of the brain. MRI of the brain was performed under the clinical question of HSV1-meningoencephalitis (Figure 1). 
In the area of the mesial part of the temporal lobe, hippocampal gyrus and the corresponding part of the operculum, edematous thickening of the gyrus was visible on the right with sulcus application, low signal in T1, high in T1 and TIRM measured images, without signs of diffusion restriction. In the same area, a smaller area of high T1 and low signal was visible on the SWI measured images, which will correspond to the area of hemorrhage. A smaller area of hemorrhage was also visible within the temporal horn of the right lateral ventricle. There was no opacification of leptomeninges in postcontrast sections. In the white matter of both frontal lobes, several small areas of high T2 and TIRM signals, of a non-specific character, were visible. No signs of fresh ischemia, hemorrhage or tumor process were seen in the remaining brain supra and infratentorially. The ventricular system was normoponated, normal width. The subarachnoid spaces of the convexity of the brain were of adequate width. The morphological findings of the optical chiasm, cavernous sinuses, sellar and suprasellar areas, pontocerebellar angles and craniocervical junction were correct. It was concluded that the changes correspond to the changes within HSV-1 encephalitis with a smaller area of hemorrhage.

When herpetic encephalitis was diagnosed, therapy with acyclovir $3 \times 10 \mathrm{mg} / \mathrm{kg}$ i.v. for three weeks was started, with mandatory monitoring of renal parameters due to nephrotoxicity and with antiulcer and antithrombotic prophylaxis. During the hospitalization, the patient was also consulted by an endocrinologist due to elevated blood glucose.

After antiviral therapy and glycemic control, the patient was released oriented, mobile, with orderly vital parameters with the next therapy; levetiracetam $2 \times 500 \mathrm{mg}$, pantoprazole $40 \mathrm{mg}$, ramipril $2.5 \mathrm{mg}$, insulin as before. It was also indicated to do a control EEG after 14 days as well as an infectologist control with laboratory findings.

\section{DISCUSSION}

Herpes simplex virus encephalitis (HSVE) can be caused by primary and recurrent herpes simplex virus (HSV) infection [1]. In more than $70 \%$ of cases, HSVE is caused by reactivation of the latent virus in people who have been previously infected [8]. After reactivation occurs, viral particles are transported by anterograde axon transport via the olfactory and trigeminal pathways to the central nervous system (CNS). In $30 \%$ of cases, HSVE is caused by a primary infection, and the virus also reaches the CNS via the olfactory and trigeminal nerves [9].

Clinical manifestations, imaging studies and cerebrospinal fluid (CSF) analysis are the cornerstones of the diagnostic approach to HSVE [10]. The most common symptoms are fever $(90 \%)$, headache $(81 \%)$, psychiatric symptoms (71\%), convulsions (67\%), vomiting (46\%), weakness (33\%), and memory loss (24\%) [11].
Complications of HSVE can be divided into acute, including cerebral edema, intracranial hypertension, cerebral hernia and seizures or chronic, which depend on the affected areas, and may include anti-NMDA (N-methyl-Daspartate) receptor encephalitis [12]. In case of suspected HSVE, prior to lumbar puncture, urgent CT of the brain is required when one or more of the following are present: evidence of obstructively elevated intracranial pressure, Glasgow Coma Scale (GCS) $<13$, decline in GCS $>2$, focal neurological signs, abnormal posture, papillary edema, and immune-compromisation. Normal CT of the brain cannot rule out a diagnosis [3]. In immunocompetent hosts, HSVE affects regions of the brain such as the limbic system, mesial temporal and frontal regions (amygdala, hippocampus, parahypocampal gyrus, temporal uncus, insula, and cingulate gyrus) early and most severely. The putamen and basal ganglia are not usually affected [13]. In many cases, HSVE is initially monolateral, but the lesions gradually become widespread and bilateral, affecting the contralateral temporal lobe asymmetrically. Sometimes the occipital cortex is affected. The lesions are uneven and asymmetric. HSVE is characterized by necrotic and hemorrhagic lesions, with perivascular lymphocytic infiltration in necrotic tissues and meninges. Performing a lumbar puncture is usually the next diagnostic step during the approach of patients with possible infectious encephalopathy [14].

Usually CSF analysis shows pleocytosis, elevated protein and erythrocyte levels and normal glucose levels [15]. The HSV CSF PCR test is the gold standard for the diagnosis of HSVE, a test with $98 \%$ specificity and $94 \%$ sensitivity. CSF sampling early or late after the onset of symptoms may reduce the possibility of a positive HSV PCR result [16].

According to [17], regarding HSV PCR testing in CSF samples, it was concluded that the higher the initial viral load, the longer the PCR test remains positive during therapeutic intervention. Furthermore, if cerebrospinal fluid samples are collected early after symptoms, PCR testing may be negative. In cases of negative first results, the clinician should repeat the test after two days, and the duration of acyclovir treatment shorter than 8 days usually does not lead to PCR that the test is negative [17]. It was found that $26 \%$ of patients with confirmed HSVE had normal WBC in collected CSF samples [18]. Viral load in the pleocytosis group was higher than in the normocellular group. Viral load didn't affect the final clinical outcome. In retrospective study, Sheybani et al. documented that CSF analysis was normal in $18 \%$ of all patients with identified HSVE, while CSF HSV-1 PCR test was negative in $24 \%$ of all patients [19].

The most common radiological features in HSVE are hypodense lesions of the temporal lobes and orbitofrontal regions, sometimes with petechial hemorrhage on CT brain scan, and hypointense in $\mathrm{T} 1$ and hyperintense in $\mathrm{T} 2$ images on MR brain scan. According to [20], in patients with manifestations of encephalitis and temporal lobe 
abnormalities on MR scan, the presence of bilateral involvement of the temporal lobe and lesions outside the temporal lobe, insula or cingulate is less common in patients with HSV encephalitis. Mesial temporal sclerosis, cerebral gliomatosis and MELAS should be included in the differential diagnosis when the MR image reveals bilateral involvement of the temporal lobe [21]. Paraneoplastic limbic encephalopathy, Hurst's disease, SLE, primary CNS lymphoma, complex partial status epilepticus, and neurosyphilis should also be included. It was suggested that even when cerebrospinal fluid analysis and imaging studies do not suggest a diagnosis, a diagnosis of HSV encephalitis cannot be ruled out when a high index of clinical suspicion is present [20]. Therefore, in cases of febrile encephalopathy of unknown origin, early administration of acyclovir may be crucial for patient survival. A similar conclusion was reached after noting that 4 out of 6 patients with normal CSF analysis had a nonspecific MRI scan of the brain. PCR testing was not available at the time of diagnosis [21].

According to [22], chronic alcohol abuse, high Knaus score, and delay in the first CT or MRI scan of the brain after admission were three factors independently associated with a delay in acyclovir administration. When a clinician is confronted with a patient with severe underlying disease or chronic alcohol abuse, in a high index of suspected HSV encephalitis, even when CSF seems normal, early initiation of acyclovir is crucial for the final outcome, based on the fact that delays in acyclovir use is one of the most important prognostic factors that can change [1]. A retrospective study [23] highlights the fact that magnetic resonance imaging may be more sensitive for the diagnosis of HSV encephalitis compared to the HSV PCR test.

Based on the fact that the HSV PCR test may show negative early after the onset of clinical manifestations, the authors suggest rapid MRI and early administration of acyclovir in cases where encephalitis is highly suspected [21]. Therefore, MRI is crucial for appropriate therapeutic intervention, in accordance with the clinical picture, and reliance only on the PCR test should be avoided. In terms of prognosis, male gender, increased age, lower GCS score, and delay in initiating acyclovir $>2$ days after symptom onset are major predictors of adverse outcomes in HSV encephalitis, according to [24], normal electroencephalography predicts survival independently of other factors. In their retrospective study in patients with acute encephalitis, it was found that mortality was $29 \%$ higher in the presence of radiologically determined cerebral edema, 21\% higher when status epilepticus occurred, and 19\% higher when thrombocytopenia developed during the course of the disease [25]. Patients over the age of 65, immunocompromised and/or with comorbidities were at higher risk of death. The mortality rate in untreated patients is approximately $70 \%$, and normal neurological function is not restored in $97 \%$ of surviving patients with sequelae [3].

\section{CONCLUSION}

HSVE is a rare disease but with severe neurological complications and high mortality. It's necessary to do the diagnostic methods as soon as possible, especially if neurological symptoms begin to occur during high fever. The treatment of choice is acyclovir therapy.

Author contributions: All authors have sufficiently contributed to the study, and agreed with the results and conclusions.

Funding: No funding source is reported for this study.

Declaration of interest: No conflict of interest is declared by authors.

\section{REFERENCES}

1. Tyler KL. Herpes simplex virus infections of the central nervous system: Encephalitis and meningitis, including Mollaret's. Herpes. 2004;11(Suppl 2):57A-64A.

2. Bradshaw MJ, Venkatesan A. Herpes simplex virus-1 encephalitis in adults: Pathophysiology, diagnosis, and management. Neurotherapeutics. 2016;13(3):493-508. doi: 10.1007/s13311-016-0433-7.

3. Venkatesan A, Tunkel AR, Bloch KC, et al. Case definitions, diagnostic algorithms, and priorities in encephalitis: Consensus statement of the international encephalitis consortium. Clin Infect Dis. 2013;57(8):1114-28. doi: 10.1093/cid/cit458.

4. Sili U, Kaya A, Mert A, HSV Encephalitis Study Group. Herpes simplex virus encephalitis: clinical manifestations, diagnosis and outcome in 106 adult patients. J Clin Virol. 2014;60(2):112-8. doi: 10.1016/j.jcv.2014.03.010

5. Stahl JP, Mailles A. Herpes simplex virus encephalitis update. Curr Opin Infect Dis. 2019;32(3):239-43. doi: 10.1097/QCO.0000000000000554.

6. Bewersdorf JP, Koedel U, Patzig M, et al. Challenges in HSV encephalitis: Normocellular CSF, unremarkable CCT, and atypical MRI findings. Infection. 2019;47(2):267-73. doi: 10.1007/s15010-018-1257-7.

7. Bell DJ, Suckling R, Rothburn MM, et al. Management of suspected herpes simplex virus encephalitis in adults in a UK teaching hospital. Clin Med. 2009;9(3):231-5. doi: 10.7861/clinmedicine.9-3-231.

8. Raschilas F, Wolff M, Delatour F, et al. Outcome of and prognostic factors for herpes simplex encephalitis in adult patients: results of a multicenter study. Clin Infect Dis. 2002;35(3):254-60. doi: 10.1086/341405.

9. Kennedy PGE, Chaudhuri A. Herpes simplex encephalitis. J Neurol Neurosurg Psychiatry. 2002;73(3):237-8. doi: 10.1136/jnnp.73.3.237.

10. Whitley RJ, Gnann JW. Viral encephalitis: Familiar infections and emerging pathogens. Lancet. 2002;359(9305):507-13. doi: 10.1016/S01406736(02)07681-X 
11. Kumar R. Understanding and managing acute encephalitis. F1000Res. 2020;9:F1000 Faculty Rev-60. doi: 10.12688/f1000research.20634.1.

12. Frantzidou F, Kamaria F, Dumaidi K, Skoura L, Antoniadis A, Papa A. Aseptic meningitis and encephalitis because of herpesviruses and enteroviruses in an immunocompetent adult population. Eur J Neurol. 2008;15(9):995-7. doi: $10.1111 /$ j.14681331.2008.02233.x

13. Enzmann DR, Ranson B, Norman D, Talberth E. Computed tomography of herpes simplex encephalitis. Radiology. 1978;129(2):419-25. doi: 10.1148/129.2.419.

14. Granerod J, Davies NWS, Mukonoweshuro W, et al. Neuroimaging in encephalitis: Analysis of imaging findings and interobserver agreement. Clin Radiol. 2016;71(10):1050-8. doi: 10.1016/j.crad.2016.03.015.

15. Hrishi AP, Sethuraman M. Cerebrospinal fluid (CSF) analysis and interpretation in neurocritical care for acute neurological conditions. Indian J Crit Care Med. 2019;23(Suppl 2):S115-9. doi: 10.5005/jp-journals10071-23187.

16. Rawal G, Yadav S, Wani UR, Ambastha AK. HSV encephalitis with normal CSF-A case report with review of literature. J Clin Diagn Res. 2015;9(12):OD06-7. doi: 10.7860/JCDR/2015/16999.6966.

17. Ziyaeyan M, Alborzi A, Haghighi AB, Jamalidoust M, Moeini M, Pourabbas B. Diagnosis and quantitative detection of HSV DNA in samples from patients with suspected herpes simplex encephalitis. Braz J Infect Dis. 2011;15(3):211-4. doi: 10.1016/s1413-8670(11)70177-5.
18. Saraya AW, Wacharapluesadee S, Petcharat S, et al. Normocellular CSF in herpes simplex encephalitis. BMC Res Notes. 2016;9:95. doi: 10.1186/s13104-016-1922-9.

19. Sheybani F, Arabikhan HR, Naderi HR. Herpes simplex encephalitis (HSE) and its outcome in the patients who were admitted to a tertiary care hospital in Mashhad, Iran, over a 10-year period. J Clin Diagn Res. 2013;7(8):1626-8. doi: 10.7860/JCDR/2013/5661.3221.

20. Chow FC, Glaser CA, Sheriff $H$, et al. Use of clinical and neuroimaging characteristics to distinguish temporal lobe herpes simplex encephalitis from its mimics. Clin Infect Dis. 2015;60:1377-83. doi: 10.1093/cid/civ051.

21. Jayaraman K, Rangasami R, Chandrasekharan A. Magnetic resonance imaging findings in viral encephalitis: A pictorial essay. J Neurosci Rural Pract. 2018;9(4):556-60. doi: 10.4103/jnrp.jnrp_120_18.

22. Zahr NM, Pfefferbaum A. Alcohol's effects on the brain: Neuroimaging results in humans and animal models. Alcohol Res. 2017;38(2):183-206.

23. Archibald LK, Quisling RG. Central nervous system infections. In: Layon AJ, Gabrielli A, Friedman WA, eds. Textbook of neurointensive care. Springer, 2013:427-517. doi: 10.1007/978-1-4471-5226-2_22.

24. Kim YS, Jung KH, Lee ST, et al. Prognostic value of initial standard EEG and MRI in patients with herpes simplex encephalitis. J Clin Neurol. 2016;12(2):224-9. doi: 10.3988/jcn.2016.12.2.224.

25. Thakur KT, Motta M, Asemota AO, et al. Predictors of outcome in acute encephalitis. Neurology. 2013;81(9):793-800. doi: 10.1212/WNL. 0b013e3182a2cc6d. 